\title{
TOXICIDADE AGUDA DO INSETICIDA PARATION METÍLICO E DO BIOPESTICIDA AZADIRACTINA DE FOLHAS DE NEEM (Azadirachta indica) PARA ALEVINO E J UVENIL DE PACU (Piaractus mesopotamicus)
}

\begin{abstract}
Os objetivos deste trabalho foram calcular a CL (I) ${ }_{50-96 h}$ do inseticida organofosforado paration metílico e do biopesticida azadiractina para alevinos e juvenis de pacu ( $P$. mesopotamicus). Também pretendeu-se avaliar o efeito do peso corpóreo sobre a toxicidade aguda do paration metílico e do neem para o pacu, bem como classificar o risco ambiental do uso de paration metílico e do neem para o controle de parasitas e patógenos de pacu. Foram realizados dois experimentos, em condições laboratoriais, para a determinação da concentração letal (CL (I) $\left.{ }_{50-96 h}\right)$. A CL (I) ${ }_{50-96 h}$ calculada do paration metílico foi de $3,97 \mathrm{mg} / \mathrm{L}$ para os alevinos e de $9,89 \mathrm{mg} / \mathrm{L}$ para os juvenis. Para a azadiractina foi de $1,20 \mathrm{mg} / \mathrm{L}$ para os alevinos e de $1,18 \mathrm{mg} / \mathrm{L}$ para os juvenis. As concentrações de $1,0 \mathrm{mg} / \mathrm{L}$ de paration metílico para os alevinos e de $7,5 \mathrm{mg} / \mathrm{L}$ para os juvenis e as de 0,29 e $0,59 \mathrm{mg} / \mathrm{L}$ de azadiractina não provocaram mortalidade nos animais expostos e podem ser utilizadas como referência em estudos de controle de parasitas em pacu. O paration metílico foi menos tóxico para os alevinos e para os juvenis de pacu do que a azadiractina, indicando a necessidade de cuidados com a utilização de extratos aquosos de neem no ambiente aquático.
\end{abstract}

PALAVRAS-CHAVE: PARATION METílICO; AZADIRACTINA; TOXICIDADE AGUDA; Piaractus mesopotamicus.

* Pós-graduando do Curso de Doutorado em Aqüicultura de Águas Continentais, Centro de Aqüicultura, Universidade Estadual Paulista (CAUNESP/UNESP), Campus de Jaboticabal (e-mail: cruzcl@yahoo.com).

** Doutor em Agronomia, Produção Vegetal, Professor do Departamento de Fitossanidade da Faculdade de Ciências Agrárias e Veterinárias, UNESP, Campus de Jaboticabal (e-mail: joaquim@fcav.unesp.br).

*** Doutor em Química Analítica, Professor do Departamento de Química, Faculdade de Ciências, UNESP, Campus de Bauru. 


\section{INTRODUÇÃO}

A avaliação ecotoxicológica é importante para o controle, regulamentação e classificação de novas substâncias tóxicas quanto ao potencial de risco ambiental. A identificação do perigo e a avaliação da relação dose-resposta são etapas iniciais no processo de avaliação da toxicidade ou de risco ambiental (USEPA, 2002). O interesse pelos efeitos que as substâncias tóxicas provocam na saúde dos peixes tem aumentado, devido ao desenvolvimento da piscicultura em regiões em que são realizadas atividades industriais ou agrícolas. Essas atividades geram resíduos que podem atingir as bacias hidrográficas e expor os peixes à altas concentrações de substâncias tóxicas (PARMA de CROUX, LOTESTE e CAZENAVE, 2002).

Os inseticidas organofosforados são utilizados, de modo geral, na piscicultura de forma direta para o controle de odonatas, copépodes e monogenéticos (NOGA, 1996). O paration metílico, inseticida que pertence a esse grupo químico, é utilizado no Brasil em tanques de piscicultura para controlar larvas de insetos aquáticos predadores de larvas de peixes (SILVA et al., 1993). Constitui um dos inseticidas mais empregados para o controle de predadores aquáticos em tanques de produção e na preparação de viveiros de recepção de larvas de peixe para maximizar a produção de rotíferos (SENHORINI et al., 1991). A aplicação de inseticidas pode provocar efeitos tóxicos agudos nos peixes e resultar em altos riscos de intoxicação (RODRIGUES et al. 1997).

O estudo da toxicidade aguda em peixes pode auxiliar na avaliação do risco ambiental e na determinação de doses seguras para o controle de parasitas em piscicultura. Estudos sobre a toxicidade aguda ( $\left.\mathrm{CL}(\mathrm{I})_{50-96 \mathrm{~h}}\right)$ de inseticidas organofosforados nos organismos aquáticos foram realizados com gusation A em Penaeus monodon (BATICADOS e TENDENCIA, 1991), com paration metílico em Daphnia magna (CASALDERREY, FERRANDO e ANDREU-MOLINER, 1994), com paration em Ankistrodesmus falcatus, Moina macrocopa e Oreochromis hornorum (MARTINEZ-TABCHE et al., 1994), em Gambusia affinis e Notropis ludibundis (WALTON, BROWN e LYDY, 1997), com clorpirifós, paration e paration metílico em Gambusia affinis (BOONE e CHAMBERS 1996), com clorpirifós, fenubucarb, monocrotofós e paration metílico em Oreochromis mossambicus (CALUMPANG et al. 1997), em larvas, pós-larvas e adultos de Homarus americanus com azametifós (BURRIDGE et al. 1999), e com monocrotofós em Prochilodus lineatus (PARMA de CROUX, LOTESTE e CAZENAVE, 2002).

Na tentativa de minimizar os efeitos causados pela alta toxicidade dos agrotóxicos no ambiente aquático, o biopesticida neem (cujo principal princípio ativo é a azadiractina) pode ser testado como alternativa menos impactante que os inseticidas organofosforados. O neem apresenta grande eficiência no controle de patógenos e pode resultar em menor impacto ao ambiente aquático. Segundo SCHAFF et al. (2000) e GOVINDACHARI (1992) existem mais de 300 compostos químicos isolados das diferentes partes da planta de neem, sendo a azadiractina o mais ativo.

A azadiractina da planta de neem pode ser utilizada como repelente de insetos, inseticida, produto de higiene e na medicina (VAN DER NAT et al., 1991). Estudos de toxicidade aguda da azadiractina em organismos aquáticos foram realizados em Daphnia pulex (GOKTEPE e PLHAK, 2002), em seis espécies de macro invertebrados aquáticos (DUNKEL e RICILARDS, 1998), em juvenil de salmão do pacífico (Onchorhynchus tshawytscha e O. kisutchi) e truta (O. mykiss) (WAN et al., 1996), e em molusco Lymnea acuminata (SINGH, SINGH e SINGH, 1996).

O pacu (Piaractus mesopotamicus), família Characidae e subfamília Serrasalminae (Holmberg, 1887), constitui espécie endêmica da bacia do Paraná-Paraguai. De importância comercial, representa uma das espécies de peixe mais cultivadas na região neotropical (SAINT-PAUL, 1986). Trata-se de peixe onívoro, rústico, precoce, com carne saborosa e ótima taxa de crescimento, sendo considerado excelente para o cultivo em sistemas intensivos (SILVA, 1985). Por essas razões, o pacu destaca-se como espécie importante para ser utilizada como organismo-teste em estudos de toxicidade aguda de agrotóxicos. As normas do IBAMA (1987) e da CETESB (1999) recomendam a utilização de espécies de peixes pertencentes à família Characidae. 
Os objetivos deste trabalho foram calcular a $\mathrm{CL}(\mathrm{I})_{50-96 \mathrm{~h}}$ do inseticida organofosforado paration metílico e do biopesticida azadiractina para alevinos e juvenis de pacu ( $P$. mesopotamicus). Também pretendeu-se avaliar o efeito do peso corpóreo sobre a toxicidade aguda do paration metílico e do neem para o pacu, bem como classificar o risco ambiental do uso de paration metílico e do neem para o controle de parasitas e patógenos de pacu.

\section{MATERIAL E MÉTODOS}

Os peixes utilizados neste trabalho são provenientes do Laboratório de Nutrição de Organismos Aquáticos do Centro de Aqüicultura da Universidade Estadual Paulista (UNESP), Campus de Jaboticabal. Os experimentos foram conduzidos na sala de bioensaios do Laboratório de Ecotoxicologia dos Agrotóxicos e Saúde Ocupacional, do Departamento de Fitossanidade, da Faculdade de Ciências Agrárias e Veterinárias, UNESP, Campus de Jaboticabal.

\subsection{TESTES PRELIMINARES E DE CONTROLE DE SENSIBILIDADE}

Nos testes preliminares com paration metílico e com a azadiractina foram utilizadas quatro concentrações crescentes e tratamento controle, com três repetições (RAND e PETROCELLI, 1985).

Para o controle de sensibilidade dos organismos-teste foram realizados periodicamente testes de toxicidade aguda com 24 horas de duração, utilizando-se dicromato de potássio e cloreto de sódio (pureza de 99,9\%) como substâncias referência de acordo com a metodologia do IBAMA (1987).

Foram determinados, preliminarmente, os intervalos de concentração de paration metílico e de azadiractina que causam zero e $100 \%$ de mortalidade para serem utilizadas nos testes definitivos, segundo metodologias recomendadas pela APHA (1991) e pelo IBAMA (1987).

Os testes foram conduzidos no sistema estático, sem substituição e sifonagem de água durante o período de exposição (96 horas) dos peixes. Os animais foram mantidos sem alimentação, sendo utilizada nos testes a água da rede de abastecimento local, proveniente de poço semi-artesiano. Avaliou-se a mortalidade diariamente com a retirada dos peixes mortos dos recipientes. Diariamente também foram coletados de cada recipiente os dados de temperatura, $\mathrm{pH}$, amônia, oxigênio dissolvido e condutividade elétrica da água (Tabela 1).

TABELA 1 - CONDIÇÕES AMBIENTAIS E VARIÁVEIS DA ÁGUA DURANTE A REALIZAÇÃO DOS TESTES DE TOXICIDADE AGUDA DO PARATION METÍLICO E DA AZADIRACTINA PARA O PACU (Piaractus mesopotamicus)

\begin{tabular}{|c|c|c|c|c|c|c|c|}
\hline & $\begin{array}{l}\text { Temperatura } \\
\text { da Sala de } \\
\text { Ensaio ("C) }\end{array}$ & $\begin{array}{l}\text { Temperatura } \\
\text { da Atgua ("C) }\end{array}$ & pH & $\begin{array}{r}\text { OxigC } \\
\text { dissol } \\
(\mathrm{mg} / \\
\end{array}$ & $\begin{array}{l}\text { CFio } \\
\text { Ivido } \\
\text { /L) }\end{array}$ & $\begin{array}{c}\text { Conduti- } \\
\text { vidade } \\
\text { (mS/cm) }\end{array}$ & $\begin{array}{c}\text { Am nia } \\
(\mathrm{g} / \mathrm{L})\end{array}$ \\
\hline $\begin{array}{l}\text { paration } \\
\text { met lico }\end{array}$ & $28-0,5$ & $26-0,5$ & $7,20-7,40$ & 8,90 & 9,00 & 0,165 & 128 \\
\hline azadiractina & $27-07$ & $26,5-0,5$ & $7,20-7,45$ & 7,15 & 7,67 & 0,178 & 156 \\
\hline $\begin{array}{l}\text { dicromato de } \\
\text { pot/Assio p.a. }\end{array}$ & $27-1,0$ & $25,5-1,0$ & $7,15 \quad 7,40$ & 7,83 & 7,90 & 0,195 & 145 \\
\hline $\begin{array}{l}\text { cloreto de } \\
\mathrm{s} \text { dio p.a. }\end{array}$ & $28-0,5$ & $26,5-1,0$ & $7,00 \quad 7,49$ & 8,10 & 8,30 & 0,150 & 178 \\
\hline
\end{tabular}


As condições ambientais recomendadas pelo IBAMA (1987) foram seguidas, exceto a temperatura da sala de ensaio. Essa, foi mantida entre 27 e $28^{\circ} \mathrm{C}$ para melhor conforto térmico do pacu (P. mesopotamicus) conforme SILVA (1985).

Os valores de CL (I) ${ }_{50-96 h}$ foram calculados pelo método Trimmed Spearman-Karber (HAMILTON, RUSSO e THURSTON, 1977).

\subsection{DETERMINAÇÃO DA TOXICIDADE AGUDA (CL (I) $\left.{ }_{50-96 \mathrm{H}}\right)$ DO PARATION METÍLICO}

Para a determinação da toxicidade aguda do paration metílico foram utilizados dois grupos de peixes: alevinos com peso entre 1 e $2 \mathrm{~g}$ e juvenis com peso entre 50 e $70 \mathrm{~g}$. Em todos os ensaios manteve-se a densidade máxima de $1 \mathrm{~g} / \mathrm{L}$ de água conforme as metodologias da APHA (1991) e da CETESB (1999).

Os peixes foram previamente aclimatados por dez dias, segundo recomendações do IBAMA (1987) e de MURTY (1988). Realizou-se a aclimatação em caixa de amianto de $250 \mathrm{~L}$, com sistema de aeração contínuo promovido por bombas de ar. Nesse período, os animais foram alimentados ad libinum com ração comercial uma vez ao dia.

Os alevinos de pacu foram expostos a seis concentrações crescentes de paration metílico e ao tratamento controle, com quatro repetições e cinco peixes por repetição $(0 ; 1,0 ; 2,5 ; 5,0 ; 7,5 ; 10,0$ e $12,5 \mathrm{mg} / \mathrm{L})$. Os animais juvenis foram expostos às concentrações de $0 ; 7,5 ; 9,0 ; 10,5 ; 12 ; 13,5 \mathrm{e}$ $15 \mathrm{mg} / \mathrm{L}$ com quatro repetições e cinco peixes por repetição.

\subsection{DETERMINAÇÃO DE AZADIRACTINA NO EXTRATO AQUOSO DE FOLHAS DE NEEM}

Para a determinação da azadiractina presente nas folhas de neem foi preparada suspensão, contento $10 \mathrm{~g}$ de folhas secas e moídas de Azadirachta indica por litro de água. Após homogeneização da suspensão, essa permaneceu em repouso por 24 horas. Filtrou-se a suspensão em papel de filtro comum e determinou-se a concentração de azadiractina presente no extrato em cromatografia a líquido de alta eficiência (CLAE). Utilizou-se cromatógrafo marca Varian, Modelo 2510, equipado com bomba recíproca, detector de ultravioleta de comprimento de onda variável (Varian modelo 2550), com comprimento de onda ajustado em $217 \mathrm{~nm}$ e integrador SP 4400 Chromajet (Varian Associates, Inc. Sunnyvale, CA, USA). Empregou-se coluna cromatográfica de superfície interna de fase reserva ISRP$\mathrm{C}_{18}(250 \mathrm{~mm} \times 2 \mathrm{~mm}$ D.I.), preparada conforme MENEZES e FÉLIX (1998). Efetuou-se a determinação da azadiractina em temperatura ambiente, com fluxo de fase móvel ajustado em $0,5 \mathrm{~mL} /$ minuto. A fase móvel utilizada foi composta pela mistura de solução aquosa de fosfato monobásico de sódio 0,05 $\mathrm{mol} / \mathrm{L}$ e acetonitrila $(63: 37 ; \mathrm{v} / \mathrm{v})$. A quantificação foi efetuada com padrão externo, com curva de calibração

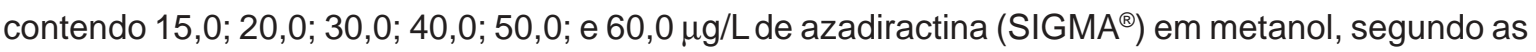
metodologias de SUNDARAM e CURRY (1993) e MENEZES et al. (2000).

\subsection{DETERMINAÇÃO DA TOXICIDADE AGUDA (CL (I) $\left.{ }_{50-96 H}\right)$ DE AZADIRACTINA}

Nos testes de toxicidade aguda para azadiractina foram utilizados alevinos de pacu com peso entre 1 e $2 \mathrm{~g}$ e juvenis com peso entre 50 e $70 \mathrm{~g}$. Em todos os ensaios foi mantida a densidade máxima de $1 \mathrm{~g} / \mathrm{L}$ de água, conforme metodologias da APHA (1991) e da CETESB (1999). Os animais foram expostos a seis concentrações crescentes de azadiractina nos extratos aquosos de folhas de neem 
e ao tratamento controle $(0 ; 0,29 ; 0,59 ; 0,88 ; 1,18 ; 1,47$ e $1,77 \mathrm{mg} / \mathrm{L})$. Os experimentos foram realizados com as mesmas concentrações para os dois grupos de peixes, pois nos testes preliminares não foi possível aumentar a concentração conforme o aumento de peso dos animais. Adotou-se o sistema estático de condução do teste, período de exposição de 96 horas, sem alimentação dos peixes. A avaliação da mortalidade foi realizada diariamente com a retirada dos indivíduos mortos dos recipientes.

\section{RESULTADOS E DISCUSSÃO}

\subsection{PARATION METÍLICO}

A equação linear que representa a relação dose-resposta e CL $(\mathrm{I})_{50-96 \mathrm{~h}}$ do paration metílico calculada para os alevinos e para os juvenis de P. mesopotamicus estão apresentadas na Tabela 2.

TABELA 2 - EQUAÇÕES LINEARES DOSE-RESPOSTA, $\mathbf{R}^{2}$ E CL (l) ${ }_{50-96 h}(\mathrm{mg} / \mathrm{L})$ DO PARATION METÍLICO E DA AZADIRACTINA PARA OS ALEVINOS E JUVENIS DE PACU (P. mesopotamicus)

\begin{tabular}{c|c|c|c|c}
\hline & \multicolumn{2}{|c|}{ Alevino } & \multicolumn{2}{c}{ Juvenil } \\
\cline { 2 - 5 } & Paration met lico & Azadiractina & Paration met lico & Azadiractina \\
\hline $\begin{array}{c}\text { Equa } \stackrel{a}{\circ} 0 \\
\text { Linear }\end{array}$ & $y=8,18 x+7,20$ & $y=101,96 x \quad 31,12$ & $y=12,38 x \quad 89,28$ & $y=99,07 x \quad 31,11$ \\
\hline$R^{2}$ & 0,958 & 0,968 & 0,965 & 0,921 \\
\hline $\mathrm{CL}(\mathrm{I})_{50-96 h}$ & 3,97 & 1,30 & 9,89 & 1,18 \\
\hline
\end{tabular}

As porcentagens de mortalidade de alevinos e juvenis durante a determinação da CL (I) ${ }_{50-96 h}$ do paration metílico estão apresentadas na Tabela 3.

TABELA 3 - MORTALIDADE (\%) DE ALEVINOS E JUVENIS DE PACU NAS DIFERENTES CONCENTRAÇÕES DE PARATION METÍLICO E DE AZADIRACTINA DURANTE A DETERMINAÇÃO DA CONCENTRAÇÃO LETAL (CL (I) $)_{50-96 h}$ )

\begin{tabular}{|c|c|c|c|c|c|c|}
\hline Alevino & \multicolumn{6}{|c|}{ Concentra I es de Paration met lico (mg/L) } \\
\hline 0,0 & 1,0 & 2,5 & 5,0 & 7,5 & 10,0 & 12,5 \\
\hline 0 & 0 & 25 & 58,6 & 75 & 91,6 & 100 \\
\hline \multicolumn{7}{|l|}{ Juvenil } \\
\hline 0,0 & 7,5 & 9,0 & 10,5 & 12,5 & 13,5 & 15 \\
\hline 0 & 0 & 25 & 50 & 50 & 75 & 100 \\
\hline Alevino & \multicolumn{6}{|c|}{ Concentra I es de Azadiractina (mg/L) } \\
\hline 0,0 & 0,29 & 0,59 & 0,88 & 1,18 & 1,47 & 1,77 \\
\hline 0 & 0 & 0 & 33,3 & 33,3 & 66,6 & 100 \\
\hline juvenil & & & & & & \\
\hline 0,0 & 0,29 & 0,59 & 0,88 & 1,18 & 1,47 & 1,77 \\
\hline 0 & 0 & 25 & 25 & 50 & 75 & 100 \\
\hline
\end{tabular}


O aumento de 2,5 vezes no valor da CL (I) ${ }_{50-96 h}$ calculada para os juvenis em relação a dos alevinos pode ser atribuída à diferença de sensibilidade ao agrotóxico, que os animais apresentam em diferentes fases da vida, e ao maior desenvolvimento do sistema enzimático de destoxificação no fígado dos animais juvenis (MOTOYAMA et al. 1991). LARINI (1987) e HAYES e LAWS (1991) citam que, geralmente, a toxicidade aguda dos compostos químicos é expressa em mg/kg. Portanto, esperavase que seria necessário aumento na concentração do paration metílico na água para se obter a CL (I) ${ }_{50-96 h}$ dos animais com peso entre 50 e $70 \mathrm{~g}$. Por outro lado, BURRIDGE et al. (1999) verificaram que a larva de lagosta (Homarus americanus) é mais tolerante ao azametifós $\left(\mathrm{CL}_{(50-48 \mathrm{~h})}\right.$ de $\left.3,57 \mu \mathrm{g} / \mathrm{L}\right)$, que o adulto $(1,39 \mu \mathrm{g} / \mathrm{L})$. Resultados semelhantes aos de BURRIDGE et al. (1999) foram obtidos por ANGUIANO et al. (1994) para embriões e larvas de Bufo arenarum expostas ao paration. Porém, o P. mesopotamicus com peso entre $1 \mathrm{e} 2 \mathrm{~g}$ (considerado alevino) foi mais sensível ao paration metílico do que os animais com peso entre 50 e $70 \mathrm{~g}$ (Tabela 2).

Após a exposição ao paration metílico, tanto os alevinos quanto os juvenis apresentaram agitação e natação não-coordenada em todos os tratamentos. Na primeira hora após a exposição, os alevinos dos tratamentos com as concentrações de 7,$5 ; 10,0$ e $12,5 \mathrm{mg} / \mathrm{L}$ e os juvenis dos tratamentos com 10,5; 12,0; 13,5 e 15,0 mg/L apresentaram sinais claros de intoxicação, com natação rápida e errática, devido à ação do agrotóxico na inibição do sistema enzimático colinérgico. Durante a primeira hora de experimentação, os peixes desses tratamentos nadaram com a cabeça para cima ou para baixo continuamente. Esses sintomas estão de acordo com os observados por MURTY et al. (1984) para o Mystus cavasius, também exposto ao paration metílico.

A maior incidência de mortalidade ocorreu nas primeiras 24 horas de exposição. Nesse período também ocorreram mortalidades crescentes com as concentrações de 5,0; 7,5; 10,0 mg/L para os alevinos e de 9,$0 ; 10,5 ; 12,0$ e 13,5 mg/L para os juvenis. Nos tratamentos com 12,5 mg/L para os alevinos e com 15,0 mg/L para os juvenis ocorreu a morte de todos os animais.

O paration metílico mostrou-se menos tóxico para o pacu nos estádios alevino e juvenil do que o clorpirifós e o paration para o peixe mosquito (Gambusia affinis), que apresentaram concentrações letais de 0,15 e 0,35 mg/L, respectivamente (BOONE e CHAMBERS, 1996). Também foi menos tóxico que o paration para os peixes $G$. affinis e $N$. ludibundis $(3517 \mu \mathrm{g} / \mathrm{L}$ e $2041 \mu \mathrm{g} / \mathrm{L}$, respectivamente) (WALTON, BROWN e LYDY, 1997). Em relação a outros organismos aquáticos, o paration metílico igualmente foi menos tóxico para o pacu. Para o camarão tigre (Penaeus monodon), a $\mathrm{CL}_{50-48 \mathrm{~h}}$ foi de 120 g/L (BATICADOS e TENDENCIA, 1991) e para Daphnia magna de 0,07 ng/L (CASALDERREY, FERRANDO e ANDREU-MOLINER, 1994). Para larvas, pós-larvas e adultos de lagosta americana (Homarus americanus) expostos a azametifós as concentrações letais foram 3,57, 2,12 e 1,39 $\mu \mathrm{g} / \mathrm{L}$, respectivamente (BURRIDGE et al. 1999). Porém, a toxicidade do paration metílico foi maior em alevinos de pacu do que a observada com monocrotofós (5,97 mg/L) em Prochilodus lineatus (PARMA de CROUX, LOTESTE e CAZENAVE, 2002) e em juvenil do que em $G$. affinis com paration metílico (13,48 mg/L) (BOONE e CHAMBERS, 1996).

Para tilápias adultas (Oreochromus niloticus), com peso entre 57 e $98 \mathrm{~g}, \mathrm{a} \mathrm{CL}_{(50-96 h)}$ foi calculada em 2,2 mg/L para o malation (PATHIRATNE e GEORGE, 1998). Para SHA-NAN e DEFANG (1996), o malation foi considerado moderadamente tóxico para peixes com CL ${ }_{(50-96 h)}$ entre 0,25 e $15 \mathrm{mg} / \mathrm{L}$. Essa faixa de toxicidade também foi observada para o paration metílico para alevinos e juvenis de pacu. Segundo BURRIDGE et al. (1999) não ocorre diferença de letalidade para o azametifós entre as formas larvas, pós-larvas e adultas dos organismos aquáticos invertebrados.

\subsection{AZADIRACTINA}

O emprego da coluna cromatográfica ISRP-C ${ }_{18}$ permitiu a injeção diluída $(1: 100 ; \mathrm{v} / \mathrm{v})$ de $500 \mu \mathrm{L}$ do extrato aquoso de neem em metanol para a determinação da azadiractina. $O$ tempo de retenção da azadiractina foi 8,82 \pm 0,04 minutos, com fator de retenção $(K)$ de 7,48 e concentração detectada de 
$11,8 \mathrm{mg}$ de azadiractina/L de extrato aquoso, semelhante às concentrações obtidas para o extrato aquoso de sementes (GOVINDACHARI, GOPALAKRISHNAN e SURESH, 1999) e para o extrato seco de sementes (JARVIS, MORGAN e EDWARDAS, 1999).

A equação linear que representa a relação dose-reposta e a concentração letal $50 \%$ da azadiractina para alevinos e para os juvenis de $P$. mesopotamicus estão apresentadas na Tabela 2 . As porcentagens de mortalidade de alevinos e juvenis ocorrida durante a determinação da CL (I) ${ }_{50-96 h}$ da azadiractina estão apresentadas na Tabela 3. A mortalidade de 100\% dos animais alevinos e juvenis também foi constatada nas primeiras 24 horas de exposição, semelhante ao observado para o paration metílico.

Em todos os tratamentos foi verificado que na primeira hora de exposição à azadiractina, os animais permaneceram no fundo do aquário. A seguir, começaram a apresentar natação errática, movimentos rápidos do opérculo. Nos tratamentos com 1,47 e $1,77 \mathrm{mg} / \mathrm{L}$, os animais permaneceram na superfície da coluna d'água para captar oxigênio atmosférico. Esse comportamento também foi descrito por OMOREGIE e OKPANACHI (1992) para tilápias (Tilápia zilli) expostas a 0,09 a $1,56 \mathrm{mg} \cdot \mathrm{dm}^{-3}$ de azadiractina em óleo de neem.

A toxicidade aguda da azadiractina também foi avaliada por DUNKEL e RICILARDS (1998) em seis espécies de invertebrados aquáticos: Drunella grandis, D. doddsi, Skwala paralela, Brachycentrus occidentalis, B. americanus e Caecidotea intermedia. $\mathrm{A} \mathrm{CL}_{50-24 \mathrm{~h}}$ calculada variou de 1,8 a 9,2 mg/L para essas espécies. KREUTZWEISER, CAPELL e SCARR (1999) calcularam a CL ${ }_{50}$ entre 0,28 e $0,84 \mathrm{mg} / \mathrm{L}$ de azadiractina para oitos espécies de invertebrados aquáticos. A CL (I) ${ }_{50-96 h}$ calculada para o $P$. mesopotamicus mostrou-se semelhante às faixas de toxicidade aguda observadas para esses invertebrados (Tabela 2).

Segundo SRIVASTAVA e RAIDAZA (2001), a azadiractina não apresentou efeito embriofetotóxico e teratogênico em ratos e não produziu efeito morfológico, visceral e esquelético. Portanto, esses autores consideraram a azadiractina como biopesticida alternativo aos inseticidas sintéticos. SCHMUTTERER (1990) calculou a dose letal $\left(\mathrm{DL}_{50}\right)$ de $7000 \mathrm{mg} / \mathrm{kg}$ para ratos e não observou toxicidade aguda oral para patos que ingeriram 5000 a $8750 \mathrm{mg} / \mathrm{kg}$ de azadiractina. Apesar desses trabalhos, dentre outros, demonstrarem a baixa toxicidade da azadiractina para mamíferos e aves é necessário cuidado na utilização desse biopesticida no ambiente aquático. Isto porque, a toxicidade aguda mostrouse alta e poderá resultar em alto risco de contaminação ambiental. SUTTON (2002) citou que os inseticidas a base de neem (azadiractina) podem apresentar riscos ao ambiente aquático, principalmente, aos insetos aquáticos e zooplâncton, especialmente os copépodas.

A utilização do extrato de folhas de neem deve ser mais estudada, devido a alta toxicidade aguda para o $P$. mesopotamicus. Outros fatores também podem limitar a sua utilização, tais como temperatura, luz ultravioleta, pH, partes da planta utilizada na preparação do extrato, degradação e efeito residual como o descrito por SCHMUTTERER (1990).

\section{CONCLUSÃO}

O paration metílico mostrou-se menos tóxico para os alevinos e para os juvenis de pacu do que a azadiractina, indicando a necessidade de cuidados com a utilização de extratos aquosos de neem no ambiente aquático. Os sinais de intoxicações causados pelos dois produtos testados em alevinos e juvenis de pacu foram semelhantes. As concentrações de 1,0 mg/L para os alevinos e de 7,5 mg/L para os juvenis expostos ao paration metílico e 0,29 e 0,59 mg/L para os alevinos e de $0,29 \mathrm{mg} / \mathrm{L}$ para os juvenis expostos a azadiractina não provocaram mortalidade nos animais. Portanto, essas concentrações podem ser utilizadas como referência em estudos de controle de parasitas em $P$. mesopotamicus. 


\begin{abstract}
ACUTE TOXICITY OF THE INSECTICIDE METHYL PARATHION AND OF THE BIOPESTICIDE AZADIRACHTIN FROM NEEM LEAVES (Azadirachta indica) TO ALEVINE AND JUVENILE PACU (Piaractus mesopotamicus) The objectives of the present study were: to calculate the lethal concentration LC (I) ${ }_{50}$ of methyl parathion and of the biopesticide azadirachtin to alevine and juvenile pacu ( $P$. mesopotamicus). Also to determine the effect of body weight on acute toxicity of methyl parathion and of neem for pacu, as well as to classify the environmental risk of the methyl parathion and neem uses for the control of pacu parasites and pathogens. Two experiments were performed under laboratory conditions for the determination of the LC (I) ${ }_{50-96 \mathrm{~h}}$. The LC (I) ${ }_{50-96 \mathrm{~h}}$ of methyl parathion was $3.97 \mathrm{mg} / \mathrm{L}$ for alevine and $9.89 \mathrm{mg} / \mathrm{L}$ for juvenile fish. The LC (I) ${ }_{50.96}$ of azadirachtin was $1.20 \mathrm{mg} / \mathrm{L}$ for alevine and $1.18 \mathrm{mg} / \mathrm{L}$ for juvenile pacu. Concentrations of 1.0 and $7.5 \mathrm{mg} / \mathrm{L}$ of methyl parathion and of 0.29 and $0.59 \mathrm{mg} / \mathrm{L}$ of azadirachtin for alevine and juvenile pacu, respectively, did not cause mortality in the exposed animals and can be used as references in studies on the control of parasites in pacu. Methyl parathion was less toxic to alevine and juvenile pacu than azadirachtin, indicating the need for careful utilization of aqueous neem extracts in aquatic environments.
\end{abstract}

KEY-WORDS: METHYL PARATHION; AZADIRACHTIN; ACUTE TOXICITY; Piaractus mesopotamicus.

\title{
REFERÊNCIAS
}

ANGUIANO, O.L.; MONTAGNA, C.M.; CHIFFLET DE LIAMAS, M.; GAUNA, L.; PECHEN de D‘ANGELO, A.M. Comparative toxicity of parathion in early embryos and larvae of the toad, Bufo arenarum Hensel. Bull. Environ. Contam. Toxicol., New York, v. 52, p. 649-655, 1994.

APHA. American Public Health Association. Standard methods for the examination of water and wastewater. $17^{\text {th }}$ ed. Washington, 1991. p. 81-143.

BATICADOS, M.C.; TENDENCIA, E.A. Effects for gusathion A on the suvival and Shell quality of juvenile Penaeus monodon. Aquaculture, New York, v. 93, p.9-19, 1991.

BOONE, J.S.; CHAMBERS, J.E. Time course of inhibition of cholinesterase and aliesterase activities and nonprotein sulfhydryl levels following exposure to organophosphorus insecticides in mosquitofish (Gambusia affinis). Fundam. Applied Toxicol., Akron, v. 29, p. 202-207, 1996.

BURRIDGE, L.E.; HAYA, K.; ZITKO, V.; WADDY, S. The lethality of salmosan (Azamethiphos) to american lobster (Homarus americanus) larvea, poslarvae and adults. Ecotox. Environ. Safety, San Diego, v. 43, p. 165-169, 1999.

CALUMPANG, S.M.F.; MEDINA, M.J.B.; TEJADA, A.W.; MEDINA, J.R. Toxicity of chorpyrifos, fenubucarb, monocrotophos and methyl parathion to fish and frogs after a simulated overflow of paddy water. Bull. Environ. Contam. Toxicol., New York, v. 58, p. 909-914, 1997.

CASALDERREY, A.F.; FERRANDO, M.D.; ANDREU-MOLINER, E. Chronic toxicity of methylparathion to Daphinia magna: Effects on survival, reproduction and growth. Bull. Environ. Contam. Toxicol., New York, v.54, p. 4349, 1994.

CETESB. Métodos de avaliação da toxicidade de poluentes a organismos aquát
toxicidade aguda com peixes - parte I - sistema estático. São Paulo, 1999. v. 2, p. 1-29.

DUNKEL, F.V.; RICILARDS, D.C. Effect of an azadirachtin formulation on six non target aquatic macroinvertebrates. Environ. Entomol, College Park, v. 27, n. 3, p. 667-673, 1998.

GOKTEPE, I.; PLHAK L. Comparative toxicity of two Azadirachtin-based neem pesticides to Daphnia pulex. Environ. Toxicol. Chem., Houston, v. 21, n. 1, p. 31-36, 2002.

GOVINDACHARI, T.R. Chemical and biological investigatins on Azadirachta indica (the neem tree). Curr. Sci, Bangalore, v. 63, n.3, p.117-122, 1992.

GOVINDACHARI, T.R.; GOPALAKRISHNAN, G.; SURESH, G. Triterpenoidal constituents of an aqueous extract form neem kernels. Fitoterapia, Milano, v. 70, p. 558-560, 1999.

HAMILTON, M.A.; RUSSO, R.C.; THURSTON, V. Trmed Spearman-Karber method for estimating medial lethal concentrations in toxicoty bioassays. Environ. Scien. Techonol., lowa, v. 7, p. 714-719, 1977.

HAYES JR, W.J.; LAWS JR., E.R. Handbook of pesticide toxicology: general principles. San Diego: Academic Press, 1991. v. 1.

IBAMA. Avaliação da toxicidade aguda para peixes: parte D. 3: manual de testes para avaliação de ecotoxicidade de agentes químicos. Brasília, DF, 1987.

JARVIS, A.P.; MORGAN. E.D.; EDWARDAS, C. Rapid separation form neem seed extracts. Phytoc. Anal., Sussex, v. 10, p. 39-43, 1999.

KREUTZWEISER, D.P.; CAPELL, S.S.; SCARR, T.A. Acute lethal and sublethal effects of a neem-based insecticide 
on nontarget aquatic insects in stream channels. Bull. Environ. Contam. Toxicol., New York, v. 63, p. 365-371, 1999.

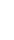

LARINI, L. Toxicologia. São Paulo: Manole, 1987. 315 p.

MARTINEZ-TABCHE L.; GALAR, C.L.; RAMIREZ, M.B.; MORALES, R.A. Parathion effect on acetylcholinesterase from fish through an artificial trophic chain: Ankitrodesmus falcatus, Moina macrocopa and Oreochromis hornorum. Bull. Environ. Contam., New York, v. 52, p. 360-366, 1994.

MENEZES, M.L.; FÉLIX, G. On line extraction and separation of bendiocarb, methomyl, methyl-parathion and pentachlorophenol pesticides from raw milk. J. Liq. Chrom. Rel. Technol., New York, v. 21, n. 18, p.2863-2871, 1998.

MENEZES, M.L.; SANCHEZ, A.; MARTINS, P.R.; GARCIA, M.A.Z.; CARDOSO, A.A.; LESSI, P.; PEREIRA, O.C.M. Determinação por injeção direta no HPLC de cocaína em amostras de urina e em amostras de papelotes de cocaína e crack. Salusvita, Bauru, v. 19, n. 2, p. 73-80, 2000.

MOTOYAMA, N.; NISHIZAWO, Y.; NAGAKURA, A.; TAKEMARA, T.; DAUTERMAN, W.C. Selective inhibition of the cytochrome P-450 dependent monooxygenaese from the liver rat and house fly abdomen. In: HODOGSON, E.; ROE, R.M.; MOTOYAMA, N. (Eds). Reviews in pesticide toxicology I. North Carolina: State University North Carolina, 1991.

MURTY, A.S. Toxicology of pesticide to fish. Boca Raton: CRC Press. 1988. v. 1.

MURTY, A.S.; RAMANI, A.V.; CHRISTOPHER, K.; RAJABHUSHANAM, B.R. Toxicity of methyl parathion and fensulfathion to the fish Mystus cavasius. Environ. Pollut. (series A), Massachusetts, v. 34, p. 37-46, 1984.

NOGA, E.J. Fish disease: diagnosis and tratament. St. Louis, Mosby: North Carolina State University, College of Veterinary Medicine, 1996. $367 \mathrm{p}$.

OMOREGIE, E.; OKPANACHI, M.A. Growth of Tilapia zilli exposed to sublethal concentrations of crude extracts of Azadirachta indica. Acta. Hydrobiol, Krakow, v. 34, n. 3, p. 281-286, 1992.

PARMA de CROUX, M.J.; LOTESTE, A.; CAZENAVE, J. Inhibition of plasma cholinesterase and acute toxicity of monocrotophos in neotropical fish Prochilodus lineatus (Pisces, Curimatidae). Bull. Environ. Contam. Toxicol., New York, v. 69, p. 356-363, 2002.

PATHIRATNE, A.; GEORGE, S.G. Toxicity of malathion to nile tilapia, Oreochromis niloticus and modulation by other environmental contaminants. Aquatic. Toxicol., Amsterdam, v. 43, p. 261-271, 1998.

RAND, G.M.; PETROCELLI, S.R. Fundamentals of aquatic toxicology. Washington: Hemisphere, 1985. 665 p.

RODRIGUES, E.D.L.; RANZANI-PAIVA, M.J.; PACHECO, F.J.; VEIGA, M.L. Efeito agudo do organofosforado Dipterex 500 (Trichlorfon) em baço de curimbatá Prochilodus scrofa (STEINDACHNER, 1881). B. CEPTA, Pirassununga, $\mathrm{n}$. 24, p. 197-203, 1997.

SAINT-PAUL, U. Potential for aquaculture for South American fish: a review. Aquaculture, New York, v. 54. p. 205-240, 1986.

SCHAAF, O.; JARVIS, A.P. ESCH, A.V.D.; GIAGNACOVO, G.; OLDHAM, N.J. Rapid and sensitive analysis of azadachtin and related triterpenoids from neem (Azadirachta indica) by high-performance liquid chromatographyatmospheric pressure chemical ionization mass spectrmetry. J. Chromat. A, Amsterdam, v.886, p. 89-97, 2000.

SCHMUTTERER, H. Properties and potencial of natural pesticides from the neem tree, Azadirachta indica. Ann. Rev. Entomol., Stanford, v. 35, p. 271-297, 1990.

SENHORINI, J.A.; FONTES, N.A.; LUCAS, A.F.B.; SANTOS J.R.S. Larvicultura do pacu Piaractus mesopotamicus Holmberg, 1887, (Pisces, Characidae) em viveiros com e sem organofosforado (folidol 600). B. CEPTA, Pirassununga, v. 4, n. 2, p.11-22, 1991.

5 SHAO-NAN, L.; DE-FANG, F. Correlation between biochemical parameters and susceptibility of freshwater fish to malathion. J. Toxicol. Environ. Heath, Washington, v. 48, p. 413-418, 1996.

SILVA, A.J. Aspectos da alimentação do pacu adulto, Colossoma mitrei (Berg, 1895) (Pisces, Characidae) no pantanal de Mato Grosso. Rio de Janeiro, 1985. 92 f. Dissertação (Mestrado em Zoologia), UFRJ.

SILVA, H.C.; MEDINA, H.S.G.; FANTA, E.; BACILA, M. Sublethal effects of the organophosphate Folidol 600 (methyl parathion) on Callichthys callichthys (Pisce: Telestei). Comp. Biochem. Physiol. C, Oxford, v. 105c, n. 2, p.197-201, 1993.

SINGH, K.; SINGH, A.; SINGH, D.K. Molluscidal activity of neem (Azadirachta indica A. Juss). J. Ethonopharm., Lausanne, v. 52, p. 35-40, 1996.

SRIVASTAVA, M.K.; RAIZADA, R.B. Assessment of embryo/fetotoxicity and teratogenicity of azadirachtin. Food. Chem. Toxicol., Oxford, v. 39, p. 1023-1027, 2001.

作 and deduos foliage, forest soils, leaf litter and stream water. J. Liq. Chromat., Florida, v. 16, n. 15, p. 3275-3290, 1993.

SUTTON, T. Environmental effects of neem-based insectisides on zooplankton communities in Forest ponds. 2002. Disponível em: <www.fnr.purdue.edu>. Acesso em: 20/03/2004. 
USEPA. United States Environmental Protection Agency. Guidelines for the health: risk assessment guidance for superfund (RAGS). 2002. Disponível em: <www.epea.gov/superfund/programs/risk/rags/ch.7>. Acesso em: 10/02/2004.

43 VAN DER NAT, J.M.; VAN DER LUIS, W.G.; SILVA, K.T.D.; LABADIE, R.P. Ethnopharmacognostical survey of Azadirachta indica A. Juss (Meliaceae). J. Ethnopharm., Lausanne, v. 35, p. 1-24, 1991.

44 WALTON, W.J.; BROWN, K.L.; LYDY, M.J. Diurnal fluctuations in toxicity in two fish species: Gambusia affinis and Notropis ludibundis. Bull. Environ. Contam. Toxicol., New York, v. 57, p. 414-421, 1997.

45 WAN, M.T.; WATTS, R.G.; ISMAN, M.B.; STRUB, R. Evaluation of acute toxicity to juvenile pacific northwest salmon of azadirachtin neem extract and neem-based products. Bull. Environ. Contam. Toxicol., New York, v. 56, p. 432-439, 1996

\section{AGRADECIMENTOS}

Os autores agradecem ao Conselho Nacional de Desenvolvimento Científico e Tecnológico (CNPq) pelo auxílio financeiro, processos n.142151/2001-7 (bolsa de doutorado do primeiro autor) e n.477882/2003-0 (Edital Universal 2002). 